\title{
Experimental and theoretical analysis of side- polished fiber optofluidic variable attenuator
}

\author{
Anna Duduś, Robert Blue, Michele Zagnoni, George Stewart and Deepak Uttamchandani \\ Centre for Microsystems and Photonics, University of Strathclyde, Glasgow G1 1XW, Scotland, UK \\ Corresponding author: anna.dudus@strath.ac.uk
}

\begin{abstract}
We report a broadband variable optical attenuator employing a side-polished single-mode optical fiber placed on an electrowetting-on-dielectric (EWOD) platform. Both experimental and theoretical analysis has been applied to this system. Experimentally, a maximum attenuation of $25 \mathrm{~dB}$ was obtained in the wavelength range between $1520 \mathrm{~nm}$ and $1560 \mathrm{~nm}$. This compares to a predicted maximum theoretical attenuation of $28 \mathrm{~dB}$.
\end{abstract}

\section{INTRODUCTION}

Single-mode fiber (SMF) variable optical attenuators (VOAs) are one of the most important elements in fibre optic communication networks ${ }^{1}$. To date, nearly all VOA are MEMS types device, that use moving fibers ${ }^{2}$, shutters $^{3}$ and mirror ${ }^{4}$ to attenuate the optical signal. Very few examples of optofluidic VOAs have been reported. One of example is a tunable microfluidic device consisting of an electrowetting-on-dielectric (EWOD) pump combined with a long period grating $(\mathrm{LPG})^{5}$, it is a narrowband VOA, with attenuation of 20 $\mathrm{dB}$ at the LPG resonant wavelength peak of $1538 \mathrm{~nm}$, and a FWHM of $10 \mathrm{~nm}$. A different type of optofluidic VOA was presented by Reza and Riza ${ }^{6}$ that is a lensed fibre gap device with a variable focus liquid lens in the gap. The achieved attenuation was up to $40 \mathrm{~dB}$. In this paper, we report a theoretical analysis of our optofluidic VOA based on a side-polished single-mode optical fiber placed on an electrowetting-on-dielectric (EWOD) platform, and demonstrate an improvement in performance from $14 \mathrm{~dB}$ previously reported ${ }^{7}$ to $25 \mathrm{~dB}$.

\section{DEVICE OPERATION}

An in-line fibre optic broadband VOA may be constructed using a side-polished SMF attached to a dielectric platform (Fig.1a). The platform is designed to translate a fluid droplet along the polished surface of the fibre using electrowetting actuation. The position of discrete $2 \mu \mathrm{L}$ and $0.3 \mu \mathrm{L}$ volume droplets on the polished surface determines how much light can be coupled out from the fibre. As illustrated in Fig 1b, if the droplet position is moved closer to the core (from 1 to 3), transmission through the fibre will progressively decrease if the fdroplet has refractive index close to the core index.

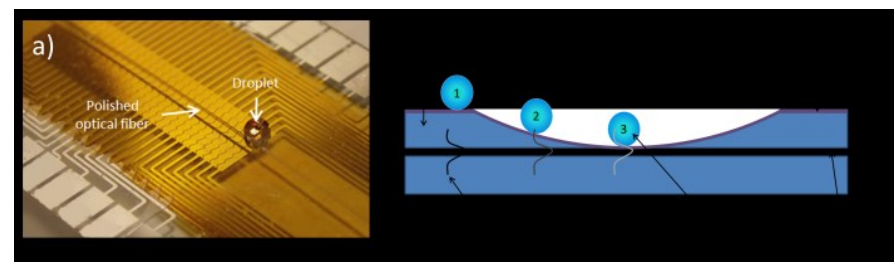

Figure 1. a) Variable optical attenuator formed from a side-polished fiber mounted on an EWOD platform. b) Illustration of the topology of a side-polished fiber

\section{THEORETICAL MODULATION}

A theoretical analysis was carried out to estimate how the guided optical power in a polished fiber will change according to changing cladding thickness, refractive index of the droplet, and the size of the interaction length of the polished region and the droplet. The output power $\mathrm{P}_{\text {out }}$ of the polished fiber can be related to the input power $\mathrm{P}_{\text {in }} \mathrm{by}^{8}$ :

$$
\frac{P_{\text {out }}}{P_{\text {in }}}=R^{\eta z}
$$

where $R$ is reflectivity, $\eta$ is the number of reflections at the upper boundary per unit length, and $\mathrm{z}$ is the propagation direction (Fig. 2). The reflectivity depends on the refractive index of the core, cladding and surrounding medium and, critically, on the remaining cladding thickness ${ }^{9}$.

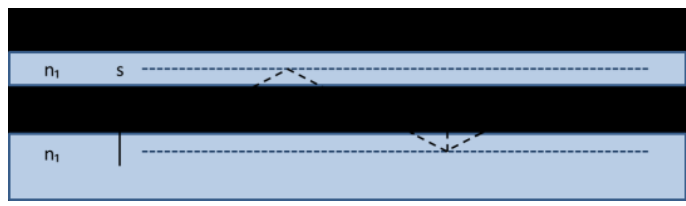

Figure 2. Schematic of the ray optics approach applied to a side-polished optical fiber. 


\section{EXPERIMENTAL CHARACTERISATION}

A square waveform of amplitude 180 volts was sequentially applied to the electrodes which translated glycerine-water solution droplets along the polished region of the fiber. A light source (EDFA) and an Optical Spectrum Analyser (Agilent 86140B) was used as a photoreceiver. Two fibers were tested. Fiber 1 was a commercial side-polished fiber from Phoenix Photonics Ltd (UK). In comparison fiber 2 was fabricated in-house to less remaining cladding. The 2 $\mu \mathrm{L}$ droplets had a measured $1.2 \mathrm{~mm}$ interaction length with polished region and three different refractive indexes were used $(1.4596,1.4602,1.4606)$. The smaller droplet of $0.3 \mu \mathrm{L}$ had an interaction length of $0.6 \mathrm{~mm}$ and a refractive index of 1.4596 . For the fiber 2 the experiment was limited to the one size of the droplet of interaction length of $1.2 \mathrm{~mm}$ but two different refractive indexes. It can be seen from figure 3 that the size of the droplet has significant influence on the results. If the interaction length decreases by half the attenuation will decrease from $7 \mathrm{~dB}$ to $4 \mathrm{~dB}$.

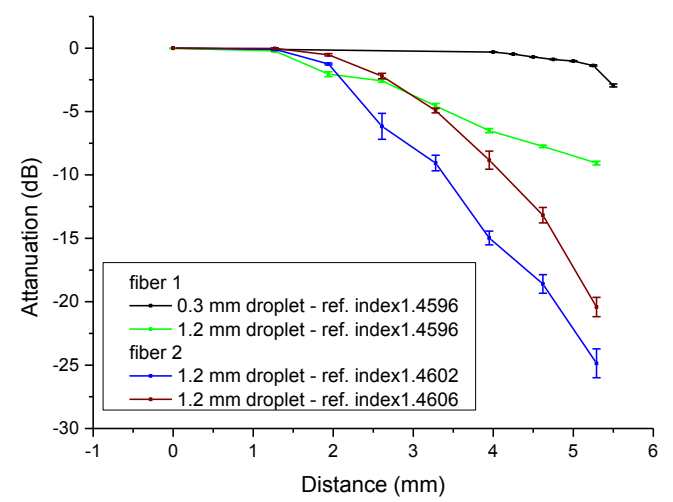

Figure 3. Experimental results for different refractive index and size of the droplet.

A theoretical analysis was performed using the physical parameters of the experiments, and is shown in figure 4. By comparing figures 3 and 4 it estimated that the remaining cladding thickness of fiber 1 and 2 were approximately $300 \mathrm{~nm}$ and $130 \mathrm{~nm}$ repectively. It can also be observed that if the refractive index is closer to the refractive index of the core (1.46) the attenuation increases from $20 \mathrm{~dB}$ to $25 \mathrm{~dB}$ (fiber 2). From the theoretical analysis the maximum attenuation that can be achieved for a refractive index of 1.4602 would be $28 \mathrm{~dB}$, for no remaining cladding. Theoretical analysis also reveals that by increasing the interaction length of the droplet with the fiber the attenuation can double from $28 \mathrm{~dB}$ to $55 \mathrm{~dB}$.

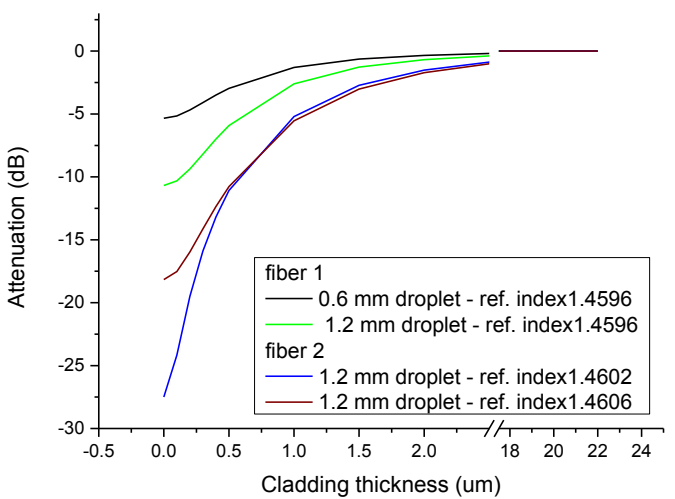

Figure 4. Theoretical results for different refractive index and size of the droplet.

\section{CONCLUSION}

We have theoretically and experimentally demonstrated an optofluidic broadband optical fiber VOA. This VOA achieved an experimental attenuation range up to 25 $\mathrm{dB}$, whilst $28 \mathrm{~dB}$ was predicted theoretically. The size of the droplet was shown to have a strong influence on the maximum attenuation that can be achieved. The attenuation can be improved up to $55 \mathrm{~dB}$ when the droplet size is doubled.

\section{REFERENCE}

${ }^{1}$ C. Lee and J. A. Yeh, J. Micro/Nanolith. MEMS MOEMS 7, 021003-1 (2008).

${ }^{2}$ A. Unamuno and D. Uttamchandani, IEEE Photonic Tech. Lett. 18, 88 (2006).

${ }^{3}$ J. C. Chiou and W. T. Lin, Opt. Commun. 237, 341 (2004).

${ }^{4}$ H. Cai, X. M. Zhang, C. Lu, A. Q. Liu, and E. H. Khoo, IEEE Photonic Tech. Lett. 17, 402 (2005).

${ }^{5}$ J. Hsieh, P. Mach, F. Cattaneo, S. Yang, T. Krupenkine, K. Baldwin and J. A. Rogers, IEEE Photonic Tech. Lett. 15, 81 (2003).

${ }^{6}$ S. A. Reza and N. A. Riza, Opt. Commun. 282, 1298 (2009).

${ }^{7}$ A. Dudus, R. Blue, M. Zagnoni, and D. Uttamchandani, "Side-polished fiber optofluidic attenuator based on electrowetting-on-dielectric actuation”, Optical MEMS \& Nanophotonics, Kanazawa, Japan, 18-22 August 2013.

${ }^{8} \mathrm{G}$ Stewart and B Culshaw, Opt. and Quant. Electron., 26, 249 (1994).

${ }^{9} \mathrm{M}$ Born and $\mathrm{E}$ Wolf, Principles of optics: electromagnetic theory of propagation, interference and diffraction of light, Chapter 1 (Cambridge University Press, $7^{\text {th }}$ edition, NY 1999). 